\title{
The disprove of the Komlos Conjecture
}

\author{
Samir Brahim Belhaouari ${ }^{1}$ and Randa AlQudah ${ }^{2}$ \\ ${ }^{1}$ Division of Information \& Computing Technology, College of Science and Engineering, Hamad Bin Khalifa \\ University; sbelhaouari@hbku.edu.qa. \\ ${ }^{2}$ Electrical and Computer Engineering, Texas A\&M University at Qatar; randa.alqudah@qatar.tamu.edu \\ Correspondence should be addressed to Samir Brahim Belhaouari: sbelhaouari@hbku.edu.qa
}

\begin{abstract}
Komlos conjecture is about the existing of a universal constant $K$ such that for all dimension $n$ and any collection of vectors $\overrightarrow{V_{1}}, \ldots, \overrightarrow{V_{n}} \in \mathbb{R}^{n}$ with $\left\|\overrightarrow{V_{l}}\right\|_{2} \leq 1$, there are weights $\varepsilon_{i} \in\{-1,1\}$ in such that

$\left\|\sum_{i=1}^{n} \epsilon_{i} \vec{V}_{l}\right\|_{\infty} \leq K(n) \leq K$. In this paper, the constant $K(n)$ is evaluated for $n \leq 5$ to be $K(2)=\sqrt{2}, K(3)=$ $\frac{\sqrt{2}+\sqrt{11}}{3}, K(4)=\sqrt{3}$, and $K(5)=\frac{4+\sqrt{142}}{9}$. For higher dimension, the function $f(n)=\sqrt{n-\left\lceil\log _{2}\left(2^{n-1} / n\right)\right\rceil}$ is found to be the lower bound for the constant $K(n)$, from where it can be concluded that the Komlos conjecture is false i.e., the universal constant $K$ does not excit because of $\lim _{n \rightarrow \infty} K(n) \geq \lim _{n \rightarrow \infty} \sqrt{\log (n)-1}=+\infty$.
\end{abstract}

Keywords: Komlos Conjecture; optimization; discrepancy theory.

\section{Introduction}

J. Komlos has made the following conjecture: For a given dimension $n$, let $K(n)$ denote the minimum value such that: for any set of $n$ Vectors $\overrightarrow{V_{1}}, \ldots, \overrightarrow{V_{n}} \in \mathbb{R}^{n}$ with $\left\|\vec{V}_{i}\right\|_{2} \leq 1$, there exists weights $\epsilon_{i}=$ +1 or -1 such that

$$
\left\|\sum_{i=1}^{n} \varepsilon_{i} \vec{V}_{i}\right\|_{\infty} \leq K(n) .
$$

Kolomos has conjected the existence of a universal constant $K$ such that $K(n) \leq K$ for all dimension $n$. The $l_{2}$ and $l_{\infty}$ norms in $\mathbb{R}^{n}$ are denoted by $\|.\|_{2}$ and $\|.\|_{\infty}$ respectively.

This conjecture was referred by Joel Spencer [8] inn 1994, where he linked kolomos Conjecture to Spencer's famous Six Standard Deviation in 1985, see [9].

The main nontrivial result known, which is due to Joel Spencer [9], is that if $k \leq n$ then $L=O(\log (L))$. The main result of D. Hajela [4] was very close to disprove the Komlos conjecture, where precisely he have proved the following theorem:

THEOREM 1. Let $f(n)$ be a function that's goes to infinity when $n$ goes to infinity with $f(n)=O(n)$ and let $0<\lambda<1 / 2$. Then form $n \geq n_{0}$ (where $n_{0}$ depends only on $n$ and $\lambda$ ) and any $A \subseteq\{1,-1\}^{n}$ with $|A| \leq 2^{n / f(n)}$, there are orthogonal vectors $x_{1}, \ldots, x_{n}$ in $R^{n}\left\|x_{i}\right\|_{2}$ for all $1 \leq i \leq n$ and such that 


$$
\left\|\varepsilon_{1} x_{1}+\cdots+\varepsilon_{n} x_{n}\right\|_{\infty} \geq \exp \left(\frac{\lambda \log \log f(n)}{\log \log \log f(n)}\right)
$$

for all $\left(\varepsilon_{1}, \ldots, \varepsilon_{n}\right) \in A$.

The previous theorem disproves the conjecture of Komlos over the set $A \subseteq\{1,-1\}^{n}$ where $|A| \leq$ $2^{n / f(n)}$.

The proof of Theorem 1 is based on certain inequalities which arise in the geometry of convex bodies [1], [10], and [2].

Komlos Conjecture is also related to discrepancy theory, paper of J.Becka and T.Fiala [6], where it states that for a global constant $K$ and for any $m \times n$ matrix $A$, whose columns are inside a unit ball, there exists a vector $X \in\{-1,+1\}^{n}$ such that $\|A X\|_{\infty} \leq K$.

The best progress in proving Komlos conjecture is a results by Banaszczyk [11] who proved the bound

$$
\min _{x \in\{-1,+1\}^{n}}\|A X\| \leq K \sqrt{\log (n)}
$$

for a global constant.

This is the best known bound for Becka-Fiala conjecture as well [5].

Discrepancy is a challenging problem that has application in geometry, data analysis, and complexity theory. The books, J. Matousek [7], B. Chazelle [ 3], and J. Beck and V.T [5], provide references for a wide array of application.

For lower dimension, The idea is to find a hypercube of minimum side of $2 k$, where all vectors formed by different combinations of the weights, $\sum_{i=1}^{n} \varepsilon_{i} \vec{V}_{i}$, should be all inside the hypercube. Also It is not hard to show that $\sqrt{n}$ is an upper bound for the constant $K$. To prove that for all vectors $\overrightarrow{V_{1}}, \ldots, \overrightarrow{V_{n}} \in \mathbb{R}^{n}$ with $\left\|\vec{V}_{l}\right\|_{2} \leq 1$, we can find $\varepsilon_{i}^{*}$ such that

$$
\left\|\sum_{i=1}^{n} \varepsilon_{i}^{*} \vec{V}_{i}\right\|_{\infty} \leq K \leq \sqrt{n},
$$

It is enough to highlight the below boulets

- We will prove first that $\left\|\sum_{i=1}^{n} \varepsilon_{i}^{*} \vec{V}_{i}\right\|_{2} \leq \sqrt{n}$, which it is a sufficient condition to prove that $\left\|\sum_{i=1}^{n} \varepsilon_{i} \vec{V}_{i}\right\|_{\infty} \leq \sqrt{n}$.

- For dimension 2: From cosine rule, we can write the following

$$
\begin{aligned}
\left\|\varepsilon_{1}^{*} \vec{V}_{1}+\varepsilon_{2}^{*} \vec{V}_{2}\right\|_{2} & =\quad\left\|\vec{V}_{1}+\frac{\varepsilon_{1}^{*}}{\varepsilon_{2}^{*}} \vec{V}_{2}\right\|_{2} \\
& =\sqrt{\left\|\overrightarrow{V_{1}}\right\|_{2}^{2}+\left\|\overrightarrow{V_{2}}\right\|_{2}^{2}-2\left\|\overrightarrow{V_{1}}\right\|_{2}\left\|\overrightarrow{V_{2}}\right\|_{2} \cos \left(\overrightarrow{V_{1}}, \overrightarrow{V_{2}}\right)} \\
& \leq \quad \sqrt{2}
\end{aligned}
$$


- If we suppose that $\left\|\sum_{i=1}^{n-1} \varepsilon_{i}^{*} \vec{V}_{i}\right\|_{2} \leq \sqrt{n-1}$, we need to prove that $\left\|\sum_{i=1}^{n-1} \varepsilon_{i}^{*} \vec{V}_{i}+\varepsilon_{n}^{*} \vec{V}_{n}\right\|_{2} \leq$ $\sqrt{n}$. Again by cosine rule, we can write the following:

$$
\begin{aligned}
& \left\|\sum_{i=1}^{n-1} \varepsilon_{i}^{*} \vec{V}_{i}+\varepsilon_{n}^{*} \vec{V}_{n}\right\|_{2} \leq \sqrt{\left\|\vec{V}_{n}\right\|_{2}^{2}+\left\|\sum_{i=1}^{n-1} \varepsilon_{i}^{*} \vec{V}_{i}\right\|_{2}^{2}} \\
& \leq \quad \sqrt{1+n-1} \\
& \leq \sqrt{n} \text {. }
\end{aligned}
$$

- From the principle of induction proof we can say that all vectors $\overrightarrow{V_{1}}, \ldots, \overrightarrow{V_{n}} \in \mathbb{R}^{n}$ with $\left\|\vec{V}_{l}\right\|_{2}$ is at most equal to 1 , we can find $\varepsilon_{i}^{*}$ such that

$$
\left\|\sum_{i=1}^{n} \varepsilon_{i}^{*} \vec{V}_{i}\right\|_{2} \leq \sqrt{n}
$$

Since $\left\|\sum_{i=1}^{n} \varepsilon_{i}^{*} \vec{V}_{i}\right\|_{\infty} \leq\left\|\sum_{i=1}^{n} \varepsilon_{i}^{*} \vec{V}_{i}\right\|_{2}$, we can conclude that the constant of Komlos has an upper bound of order $\sqrt{n}$.

We can extend the Komlos conjecture statement to the below lemma, where it summarizes very interesting properties related to special vectors, $\vec{V}_{l}^{*}, i=1, \ldots, n$, that cannot cancel each other further than $K(n)$.

Lemma: Let $C^{n}$ a set of vectors in $\mathbb{R}^{n}$ have $l_{2}$ norm at most 1 , and we denote by $V^{*}$ as a set of vectors in $\mathbb{R}^{n}$ that satisfies $V^{*}=\left\{{\overrightarrow{V_{1}}}^{*}, \ldots,{\overrightarrow{V_{n}}}^{*}\right\}=\underset{\vec{V}_{i} \in C^{n}}{\operatorname{argmax}} \min _{\varepsilon_{i}}\left\|\sum_{i=1}^{n} \epsilon_{i} \vec{V}_{l}^{*}\right\|_{\infty}$

The set $V^{*}$ satisfies the below properties:

i. For all vector $\vec{V}_{l}^{*}$ in $V^{*}$ has $l_{2}$ norm equal to $1,\left\|\vec{V}_{l}^{*}\right\|_{2}=1$.

ii. All the vertices have the same distance $l_{\infty}$, i.e.,

$$
\operatorname{Min}_{\varepsilon_{i} \in\{-1,+1\}}\left\|\sum_{i=1}^{n} \varepsilon_{i} \vec{V}_{l}^{*}\right\|_{\infty}=\operatorname{Max}_{\varepsilon_{i} \in\{-1,+1\}}\left\|\sum \varepsilon_{i} \vec{V}_{l}^{*}\right\|_{\infty} \text {. }
$$

iii. $\quad K(n)$ is strictly increasing sequence, i.e., for all integers $m>n, K(n)<K(m)$.

A tentative proof of the previous lemma will be publish soon.

The following sections are consecrated to evaluate the constant $K$ for a different dimension, the exact value of $K$ will be calculated for a dimension less or equals to 6 and a lower bound will evaluated for all dimension.

\section{The constant $\mathrm{K}$ for dimension 2}

It is obvious to say that the constant $K$ for dimension one is equal to one, and it is quite easy to calculate $K$ for dimension 2, denoted by $K(2)$. 
To find the value of (2), it is useful to analyze the parallelogram formed by four vertices centered at the origin, resulting from the four combinations of $\varepsilon_{i}$, i.e., $\mp \vec{V}_{1} \mp \vec{V}_{2}$ (see fig.1)

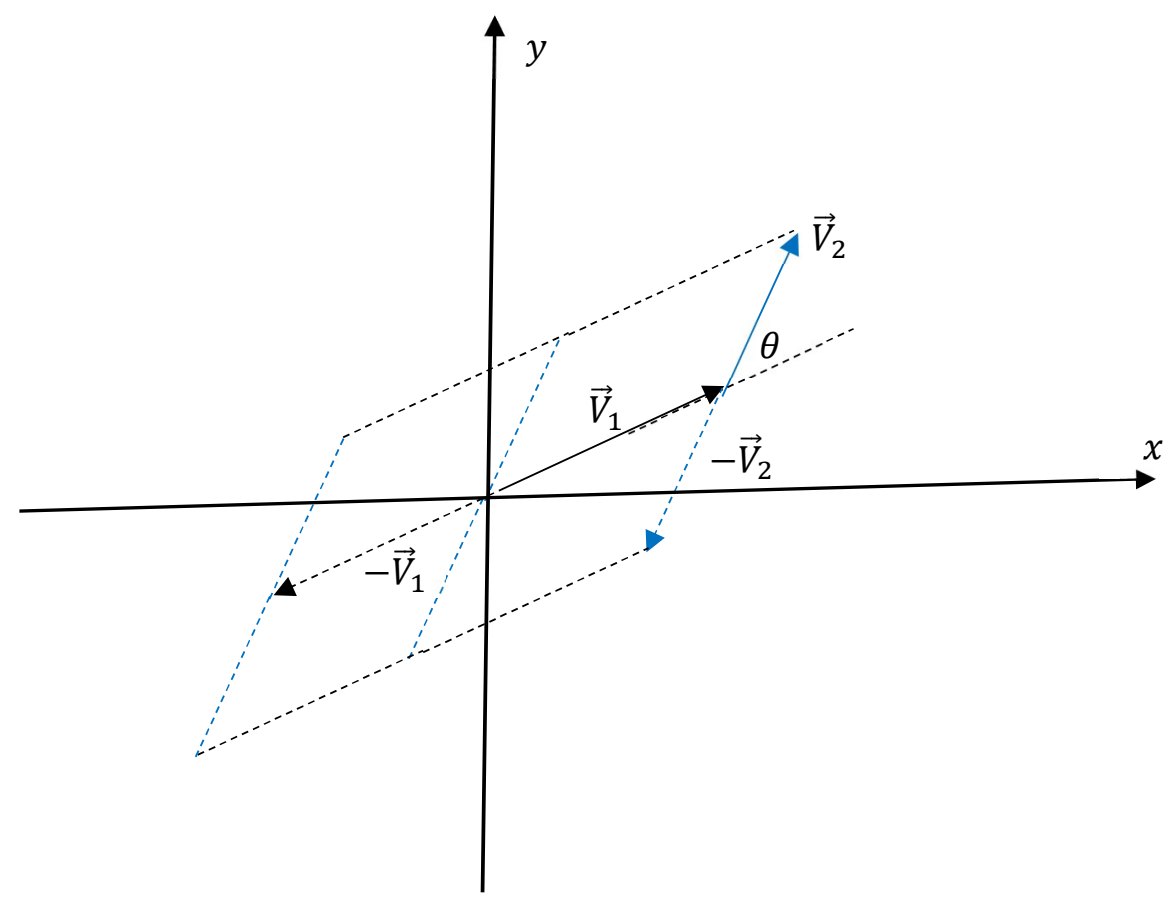

Figure 1 A parrallegram formed by four vertices, $\vec{V}_{1}+\vec{V}_{2}, \vec{V}_{1}-\vec{V}_{2},-\vec{V}_{1}+$ $\vec{V}_{2},-\vec{V}_{1}-\vec{V}_{2}$.

By using the cosine rule, we can find the length of the big and the small diagonals respectively as follows:

$$
\left\{\begin{array}{l}
L^{2}=\left\|\vec{V}_{1}\right\|_{2}+\left\|\vec{V}_{2}\right\|_{2}+2\left\|\vec{V}_{1}\right\|_{2}\left\|\vec{V}_{2}\right\|_{2} \cos (\theta) \\
l^{2}=\left\|\vec{V}_{1}\right\|_{2}+\left\|\vec{V}_{2}\right\|_{2}-2\left\|\vec{V}_{1}\right\|_{2}\left\|\vec{V}_{2}\right\|_{2} \cos (\theta)
\end{array}\right.
$$

where $\theta$ is the acute angle between the two vectors $\vec{V}_{1}$ and $\vec{V}_{2}$.

We can notice that the small diagonal $l$ has $\sqrt{2}$ as an upper bound, i.e., $l \leq \sqrt{2}$.

The two weights, $\epsilon_{1}$ and $\epsilon_{2}$, can be chosen in such away $\varepsilon_{1} \vec{V}_{1}+\varepsilon_{2} \vec{V}_{2}$ gives a small diagonals, in which it implies that for all vectors $\vec{V}_{i}$ inside the circle of center $(0,0)$ and Radius $=1$, we can find $\epsilon_{1}$ and $\epsilon_{2}$ such that $\left\|\varepsilon_{1} \vec{V}_{1}+\varepsilon_{2} \vec{V}_{2}\right\|_{\infty} \leq \sqrt{2}$.

We can prove that $K(2) \leq \sqrt{2}$ by contradiction, Assume the exitance of 4 vertices A, B, C, D outside of the square of side $2 \sqrt{2}$ as it shown in figure 2 . 


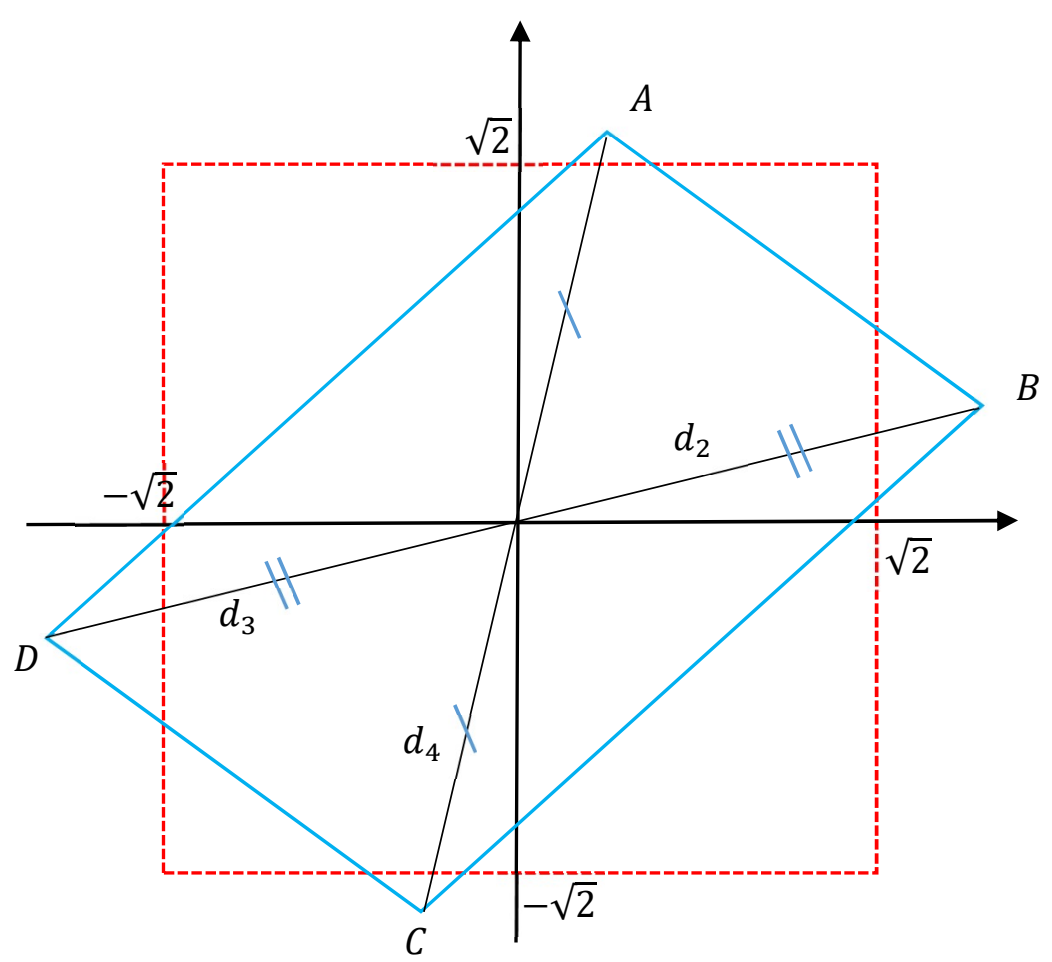

Figure $2 A B C D$ is a parrallegram with forl vertices located outside of square whose side is $\sqrt{2}$ has $\min _{i}\left(d_{i}\right) \geq \sqrt{2}$.

The possibility to have all the vertices, $\mp \vec{V}_{1} \mp \vec{V}_{2}$, outside the red square, refer to fig2, is impossible! Because it contradicts with the fact that small diagonal length is at most $\sqrt{2}$.

From previous proof, we can conclude that $K(2) \leq \sqrt{2}$, and to proof $K=\sqrt{2}$, it is enough to find a particular case where $\min _{\varepsilon_{i}}\left\|\varepsilon_{1} \vec{V}_{1}+\varepsilon_{2} \vec{V}_{2}\right\|_{\infty}=\sqrt{2}$.

If we consider two vectors as $\vec{V}_{1}=\frac{1}{\sqrt{2}}\left(\begin{array}{l}+1 \\ +1\end{array}\right)$ and $\vec{V}_{2}=\frac{1}{\sqrt{2}}\left(\begin{array}{l}-1 \\ +1\end{array}\right)$, and for all possible values of $\epsilon_{1}$ and $\epsilon_{2}$, we can calculate $\min _{\varepsilon_{i}}\left\|\varepsilon_{1} \vec{V}_{1}+\varepsilon_{2} \vec{V}_{2}\right\|_{\infty}$ as follows:

$$
\begin{aligned}
\max \left(\frac{\left|\epsilon_{1}+\epsilon_{2}\right|}{\sqrt{2}}, \frac{\left|\epsilon_{1}-\epsilon_{2}\right|}{\sqrt{2}}\right) & =\max \left(\frac{\left|1+\epsilon_{2} / \varepsilon_{1}\right|}{\sqrt{2}}, \frac{\left|1-\epsilon_{2} / \varepsilon_{1}\right|}{\sqrt{2}}\right) \\
& =\frac{2}{\sqrt{2}} .
\end{aligned}
$$

Finally, we conclude that $K(2)=\sqrt{2}$. 


\section{The constant $K$ for dimension 3}

Given the vector space $\mathbb{R}^{3}$, the span of the set $S$ of finite vectors is defined as the set of all finite linear combinations of elements of $S$

$$
\operatorname{Span}(S)=\left\{\sum_{i=1}^{k} \alpha_{i} \vec{V}_{i} ; k \in \mathbb{N}, \vec{V}_{i} \in S, \alpha_{i} \in \mathbb{R}\right\}
$$

The calculation of $K(3)$ will be splited to several cases related to different configuration of the three vectors, $\vec{V}_{1}, \vec{V}_{2}$, and $\vec{V}_{3}$ in $\mathbb{R}^{3}$.

Case1: $\vec{V}_{3} \perp \operatorname{Space}\left(\vec{V}_{2}, \vec{V}_{1}\right)=\mathrm{XY}$-plane.

As the vector $\vec{V}_{3}$ is parallel to z-axe, then without losing generality, we can write the following:

$$
\min _{\varepsilon_{i}}\left\|\vec{\varepsilon}_{3} \vec{V}_{3}+\vec{\varepsilon}_{2} \vec{V}_{2}+\vec{\varepsilon}_{1} \vec{V}_{1}\right\|_{\infty}=\min _{\varepsilon_{i}}\left\|\vec{V}_{3}+\vec{\varepsilon}_{2} \vec{V}_{2}+\vec{\varepsilon}_{1} \vec{V}_{1}\right\|_{\infty}
$$

by consequence,

$$
\min _{\varepsilon_{i}}\left\|\vec{V}_{3}+\vec{\varepsilon}_{2} \vec{V}_{2}+\vec{\varepsilon}_{1} \vec{V}_{1}\right\|_{\infty}=\operatorname{Max}\left\{\left\|\vec{U}_{3}\right\|_{2}, \min _{\varepsilon_{i}}\left\|\vec{\varepsilon}_{2} \vec{V}_{2}+\vec{\varepsilon}_{1} \vec{V}_{1}\right\|_{\infty}\right\}
$$

From previous section, we know that the constant $K(2)=\sqrt{2}$ and from the fact that $\vec{\varepsilon}_{2} \vec{V}_{2}+\vec{\varepsilon}_{1} \vec{V}_{1} \in \mathrm{XY}$ plane, we have

$$
\begin{aligned}
\left\{\left\|\vec{U}_{3}\right\|_{2}, \min _{\varepsilon_{i}}\left\|\vec{\varepsilon}_{2} \vec{V}_{2}+\vec{\varepsilon}_{1} \vec{V}_{1}\right\|_{\infty}\right\} & \leq \operatorname{Max}\left\{\left\|\vec{U}_{3}\right\|_{2}, \sqrt{2}\right\} \\
& \leq \sqrt{2}
\end{aligned}
$$

By considering $\vec{V}_{1}=\frac{1}{\sqrt{2}}\left(\begin{array}{c}+1 \\ +1 \\ 0\end{array}\right), \vec{V}_{2}=\frac{1}{\sqrt{2}}\left(\begin{array}{c}-1 \\ +1 \\ 0\end{array}\right)$, and $\vec{V}_{3}=\frac{1}{\sqrt{2}}\left(\begin{array}{c}0 \\ 0 \\ +1\end{array}\right)$, we can calculate

$$
\begin{aligned}
\left\|\sum_{i=1}^{3} \varepsilon_{i}^{*} \vec{V}_{i}\right\|_{\infty} & \left.=\max \left(\frac{\left|\varepsilon_{1}+\epsilon_{2}\right|}{\sqrt{2}}, \frac{\left|\varepsilon_{1}-\epsilon_{2}\right|}{\sqrt{2}},\left|\varepsilon_{3}\right|\right)\right) \\
& =
\end{aligned}
$$

Therefore, under the case 1 , the constant $K(3)$ is equal to $\sqrt{2}$.

Case2: $\operatorname{Span}\left(\vec{V}_{1}, \vec{V}_{2}\right)=\mathrm{XY}$-plane.

We split the vector $\vec{V}_{3}$ as follows:

$\vec{V}_{3}=\vec{V}_{31} \oplus \vec{V}_{32}$, where $\vec{V}_{31} \perp \mathrm{XY}$-plane. Without losing generality, we will consider the weight $\varepsilon_{3}$ as one value in our calculations. 
Therefore, for all vectors $\overrightarrow{V_{1}}, \overrightarrow{V_{2}}, \overrightarrow{V_{3}} \in \mathbb{R}^{3}$ with $\left\|\vec{V}_{l}\right\|_{2} \leq 1$

$$
\begin{aligned}
\min _{\varepsilon_{i}}\left\|\sum_{i=1}^{3} \varepsilon_{i} \vec{V}_{i}\right\|_{\infty} & =\min _{\varepsilon_{i}}\left\|\vec{V}_{3}+\sum_{i=1}^{2} \varepsilon_{i} \vec{V}_{i}\right\|_{\infty} \\
& =\max \left\{\left\|\vec{V}_{31}\right\|_{2}, \min _{\varepsilon_{i}}\left\|\vec{V}_{32}+\sum_{i=1}^{2} \varepsilon_{i} \vec{V}_{i}\right\|_{\infty}\right\}
\end{aligned}
$$

From previous equation, we can see that the calculation is moved from dimension 3 to dimension 2 by just calculating the following:

For all vectors $\overrightarrow{V_{1}}, \overrightarrow{V_{2}}, \overrightarrow{V_{32}} \in \mathbb{R}^{2}$ with $\left\|\vec{V}_{l}\right\|_{2} \leq 1$, the below minimum is needed to be calculated

$$
\min _{\varepsilon_{i}}\left\|\vec{V}_{32}+\sum_{i=1}^{2} \varepsilon_{i} \vec{V}_{i}\right\|_{\infty}
$$

where $\vec{V}_{32}=\left(\begin{array}{l}\alpha \\ \beta \\ 0\end{array}\right)$, and without losing generality, we can assume that $\alpha^{2}+\beta^{2} \leq 1$ and $0 \leq \alpha \leq \beta \leq 1$.

To evaluate the constant $K(3)$, the question about the possibility to have all the vertices, $\vec{V}_{32} \pm \vec{V}_{2} \pm \vec{V}_{1}$, outside the square of side $2 \sqrt{2}$, as it shown in Figure 3 , need to be checked.

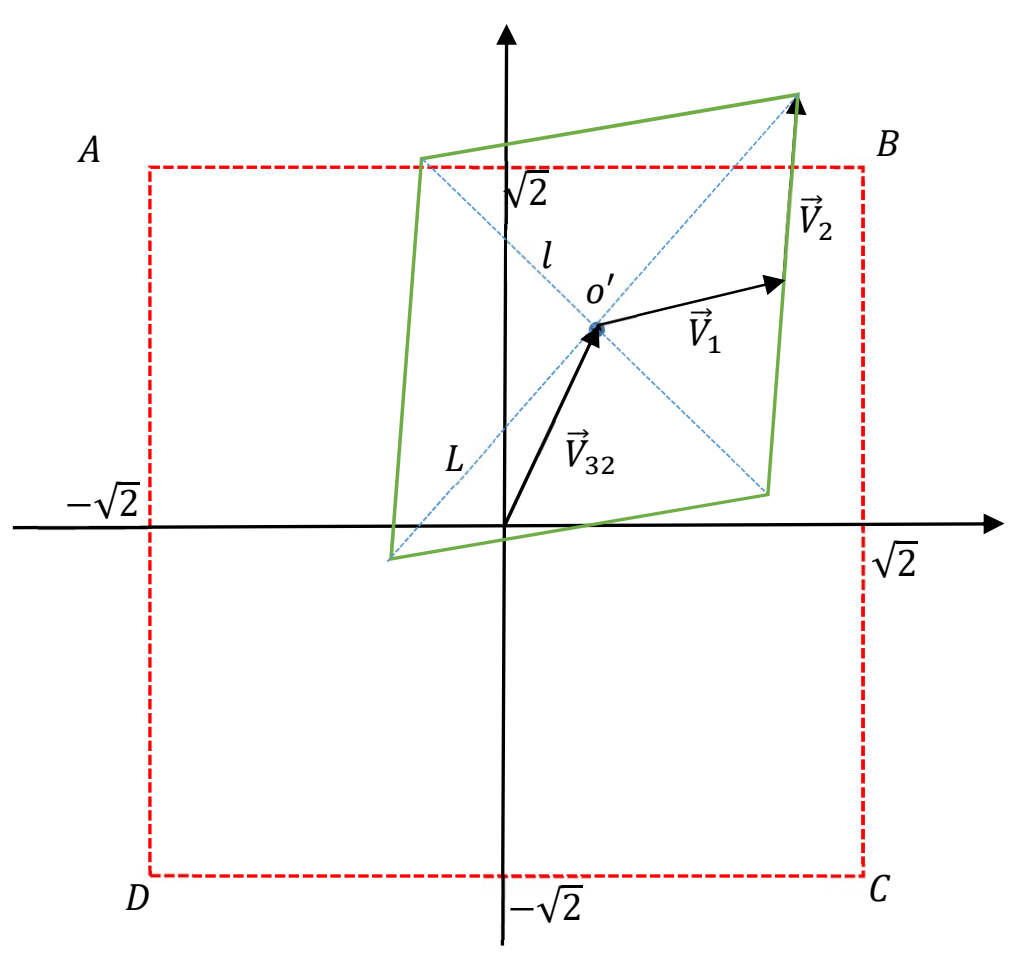

Figure 3 The vector $\vec{V}_{32}$ can be consider with slope bigger than

one, $m \geq 1$, without losing generality. Thow two distance

$l$ and $L$ are big and small diagonal respectively. 
From the Figure 4, the small diagonal, $l$, of parallogram centered at the point $O^{\prime}$ is at most equal to $\sqrt{2}$, consequently, we have to focus only on the green area, highlighted in Figure 5 , the possible location of two opposite vertices that form the two small diagonal $l$.

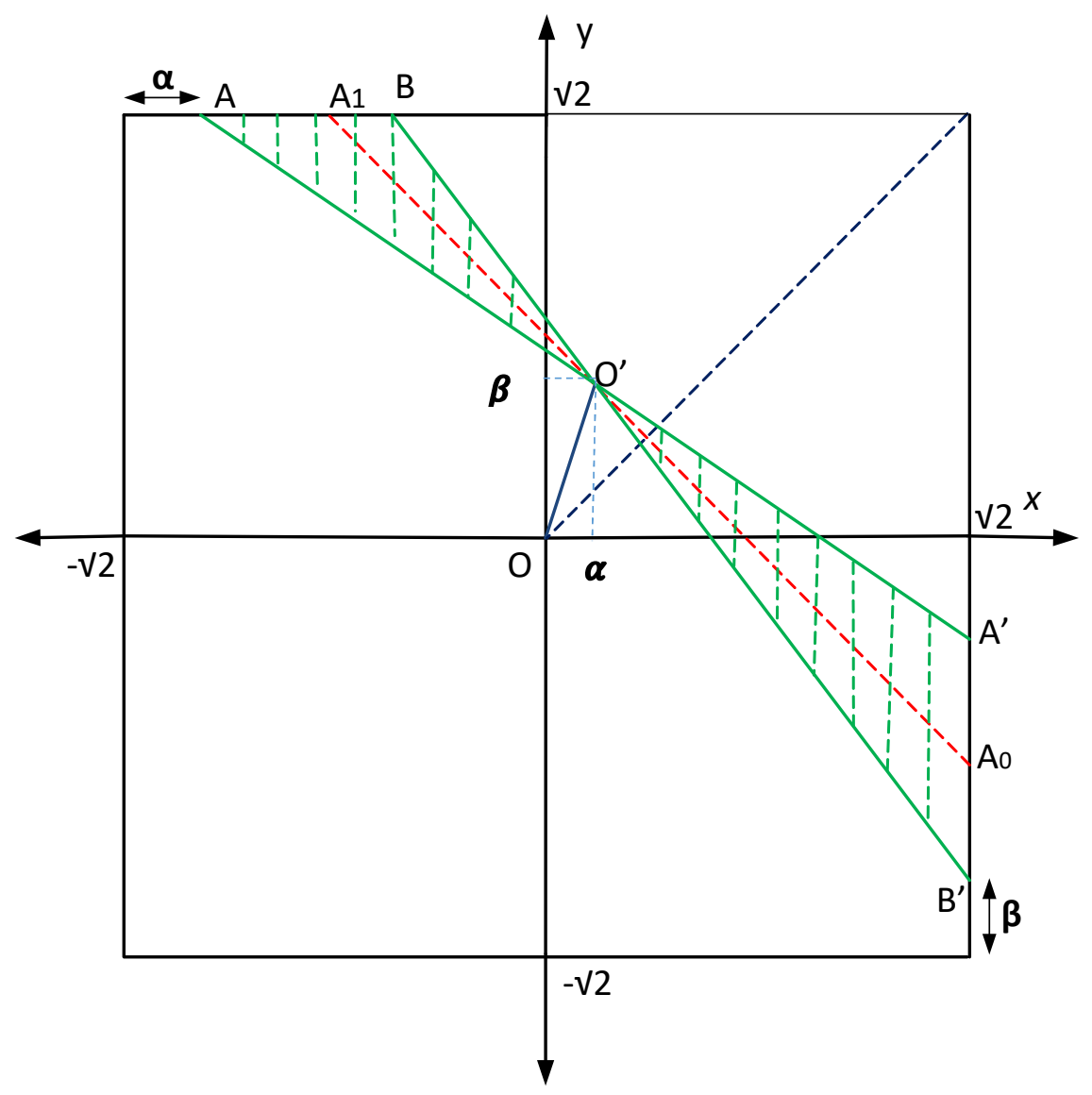

Figure 4 Green Area is the only possible location of the vertices, $\overrightarrow{V_{32}} \mp \overrightarrow{V_{1}} \mp \overrightarrow{V_{2}}$, in order to have maybe $\left\|\overrightarrow{V_{32}} \mp \overrightarrow{V_{1}} \mp \overrightarrow{V_{2}}\right\|_{\infty}>\sqrt{2}$

The distance between the point $O^{\prime}$ and the midpoint of two vertices is equals to either $\left\|\vec{V}_{1}\right\|$ or $\left\|\vec{V}_{2}\right\|$, which implies that the impossibility to have, on one side of square, two vertices outside of red square, refer to Figure 4.

This impossibility can be proved by highlighting the fact that the distance between any point inside the area $S_{1}$ and any point inside the area $S_{2}$ is bigger than or equal to 1 , see Figure 5 . 


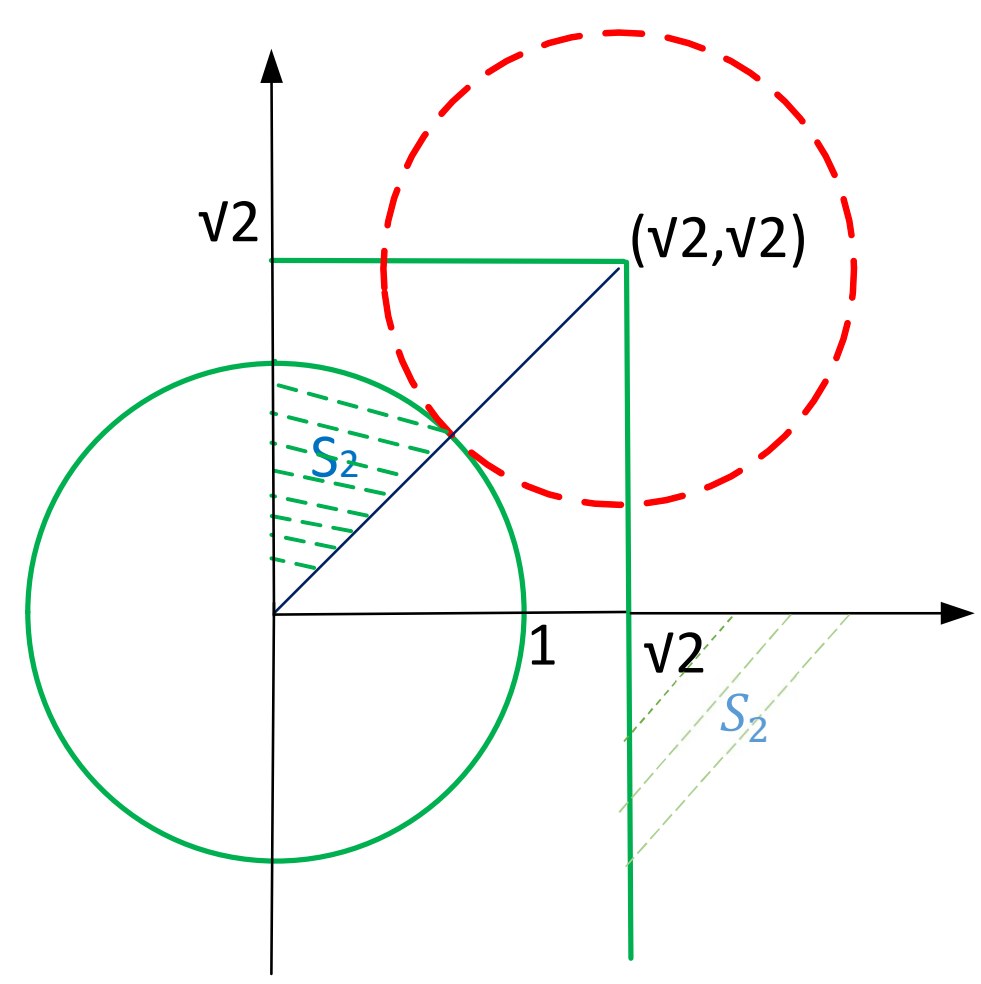

Figure 5 Distance between any point in the area $S_{1}$ and any point in the area $S_{2}$ has minimum distance of 1 ,

$$
S_{1}=\{x \geq \sqrt{2} \text { and } y \leq 0\} \text { and } S_{2}=\left\{x^{2}+y^{2} \leq 1 \text { and } 0 \leq x \leq y \leq 1\right\}
$$

Therefore, under the case 2 , the constant $K(3)$ is upper bounded by $\sqrt{2}$.

To say that $K(3)=\sqrt{2}$, we need to consider to particular example as follows:

If $\vec{V}_{1}=\frac{1}{\sqrt{2}}\left(\begin{array}{c}+1 \\ +1 \\ 0\end{array}\right), \vec{V}_{2}=\frac{1}{\sqrt{2}}\left(\begin{array}{c}-1 \\ +1 \\ 0\end{array}\right)$, and $\vec{V}_{3}=\frac{1}{\sqrt{2}}\left(\begin{array}{c}0 \\ 0 \\ +1\end{array}\right)$, then

$$
\min _{\varepsilon_{i}}\left\|\sum_{i=1}^{3} \varepsilon_{i} \vec{V}_{i}\right\|_{\infty}=\sqrt{2}
$$

Case3: General case

By symmetry, without losing generality, we can consider the weight $\epsilon_{3}=1$ in our calculations, as it is proven below 


$$
\begin{aligned}
\min _{\varepsilon_{i}}\left\|\sum_{i=1}^{3} \varepsilon_{i} \vec{V}_{i}\right\|_{\infty} & =\min _{\varepsilon_{i}}\left\|\varepsilon_{3} \sum_{i=1}^{3} \frac{\varepsilon_{i}}{\varepsilon_{3}} \vec{V}_{i}\right\|_{\infty} \\
& =\min _{\varepsilon_{i}}\left\|\vec{V}_{3}+\sum_{i=1}^{2} \frac{\varepsilon_{i}}{\varepsilon_{3}} \vec{V}_{i}\right\|_{\infty} \\
& =\min _{\varepsilon_{j}}\left\|\vec{V}_{3}+\sum_{j=1}^{2} \varepsilon_{j} \vec{V}_{i}\right\|_{\infty} .
\end{aligned}
$$

The vector $\sum_{i=1}^{3} \varepsilon_{i} \vec{V}_{i}$ will be evaluated over two perpendicular spaces, XY-plane and Z-axe, and a link between the two space will found in order to maximize the $l_{\infty}$ norm of $\left\|\sum_{i=1}^{3} \varepsilon_{i} \vec{V}_{i}\right\|_{\infty}$.

The project of the vector $\vec{V}_{i}$ over the space XY-plane is denoted by vector $\vec{U}_{i}=\operatorname{Proj}_{X Y-p l a n}\left(\vec{V}_{i}\right)$.

From case 2 , we have proven that it is not possible to have all vertices, $\vec{U}_{3} \pm \vec{U}_{2} \pm \vec{U}_{1}$, outside the square of side $2 \sqrt{2}$ centered at the origin. A question rises of the possibility to increase the $l_{\infty}$ norm beyond $\sqrt{2}$ for two vertices and compendate the $l_{\infty}$ norms of the two other vertices by $l_{\infty}$ norm over Z-axe?

To answer of the previous question, we need to find Z-coordinates of three vectors $\vec{V}_{i}$ that satisfy the following statement:

For each possible weight's vector $\left(1, \varepsilon_{1}, \varepsilon_{2}\right)$ where $\left\|\vec{U}_{3}+\sum_{j=1}^{2} \varepsilon_{j} \vec{U}_{i}\right\|_{\infty}<\sqrt{2}$ then

$$
\left\|Z_{3}+\sum_{j=1}^{2} \varepsilon_{j} Z_{i}\right\|_{\infty}=\left|Z_{3}+\sum_{j=1}^{2} \varepsilon_{j} Z_{i}\right|>\sqrt{2} \text {. }
$$

To summarize the above idea, we create an example of vectors $\vec{V}_{i}$, where $K=\left\|\sum_{i=1}^{3} \varepsilon_{i} \vec{V}_{i}\right\|_{\infty}>\sqrt{2}$, as follows:

$$
\vec{V}_{1}=\left(\begin{array}{c}
-x_{1} \\
y_{1} \\
z_{1}
\end{array}\right), \vec{V}_{2}=\left(\begin{array}{c}
-x_{2} \\
y_{2} \\
-z_{2}
\end{array}\right) \text {, and } \vec{V}_{3}=\left(\begin{array}{c}
x_{3} \\
y_{3} \\
z_{3}
\end{array}\right)
$$

where $x_{i}, y_{i}, z_{i}$ are all non-negative value with $\left\|\vec{V}_{i}\right\|_{2}=\sqrt{x_{i}^{2}+y_{i}^{2}+z_{i}^{2}} \leq 1$,

We assume the following equations

$$
\begin{gathered}
\text { if }\left\{\begin{array}{l}
\left\|\vec{V}_{3}-\vec{V}_{2}-\vec{V}_{1}\right\|_{\infty}=x_{1}+x_{2}+x_{3}=K_{1} \\
\left\|\vec{V}_{3}+\vec{V}_{2}+\vec{V}_{1}\right\|_{\infty}=y_{1}+y_{2}+y_{3}=K_{2}
\end{array}\right. \\
\text { then }\left\{\begin{array}{l}
\left\|\vec{V}_{3}-\vec{V}_{2}+\vec{V}_{1}\right\|_{\infty}=z_{1}+z_{2}+z_{3}=K_{3} \\
\left\|\vec{V}_{3}+\vec{V}_{2}-\vec{V}_{1}\right\|_{\infty}=\left|z_{3}-z_{1}-z_{2}\right|=K_{4} .
\end{array}\right.
\end{gathered}
$$

By symmetry, we can consider $K_{1}=K_{2}$ and $K_{3}=K_{4}$. 
Then the system that is needed to be solved is summarized by the following equations:

$$
\left\{\begin{array}{l}
x_{1}+x_{2}+x_{3}=K_{1} \\
y_{1}+y_{2}+y_{3}=K_{1} \\
z_{1}+z_{2}+z_{3}=K_{3} \\
z_{1}+z_{2}-z_{3}=K_{3}
\end{array}\right.
$$

From the last two equations, we conclude that

$$
z_{3}=0
$$

By symmetry, we can conclude that

$$
x_{3}=y_{3}
$$

Since $\left\|\vec{V}_{3}\right\| \leq 1$, it is convenient to increase $x_{3} \& y_{3}$ as much as we can, then the maximum value of $K_{1}$ can be found when:

$$
x_{3}=y_{3}=\frac{1}{\sqrt{2}}
$$

Therefore, the system will be simplified as follows

$$
\left\{\begin{array}{c}
x_{1}+x_{2}=K_{1}-\frac{\sqrt{2}}{2} \\
y_{1}+y_{2}=K_{1}-\frac{\sqrt{2}}{2} \\
z_{1}+z_{2}=K_{3}
\end{array}\right.
$$

Again by symmetry, we can consider the following equations:

$$
\begin{aligned}
& x_{1}=y_{1}=\alpha \\
& x_{2}=y_{2}=\beta \\
& z_{1}=z_{2}=\gamma
\end{aligned}
$$

In order to maximize $K$, we need to impose that $K_{1}=K_{3}$, then the final system that need to be solved is as follows:

$$
\left\{\begin{array}{c}
\alpha+\beta=K-\frac{\sqrt{2}}{2} \\
\gamma=\frac{K}{2} \\
\alpha^{2}+\beta^{2}+\gamma^{2} \leq 1,
\end{array}\right.
$$

the last inequality comes from the constraint that $\left\|\vec{V}_{i}\right\|_{2}=\alpha^{2}+\beta^{2}+\gamma^{2} \leq 1$.

Again, without losing generality, we can assume that $\alpha=\beta$, 
The maximize value of $K$ can be calculated by

$$
\left\{\begin{array}{c}
\alpha=\frac{K}{2}-\frac{\sqrt{2}}{4} \\
\gamma=\frac{K}{2} \\
2 \alpha^{2}+\gamma^{2}=1
\end{array}\right.
$$

So, we end up to solve the below quadratic equation

$$
2\left(\frac{K}{2}-\frac{\sqrt{2}}{4}\right)^{2}+\left(\frac{K}{2}\right)^{2}=1
$$

After simplification, we find:

$$
3 K^{2}-2 \sqrt{2} K-3=0
$$

The value $K$ is equal to $\frac{\sqrt{2}+\sqrt{11}}{3}$, then we can conclude that

$$
K(3) \geq \frac{\sqrt{2}+\sqrt{11}}{3}
$$

By using a simulation the question if $K(3)=\frac{\sqrt{2}+\sqrt{11}}{3}$ or not can be checked. A cylindrical Coordinate has been used in our simulation to check most of the cases, the possible coordinate's values of the vector $\vec{V}_{i}$ are summarized as follows:

$$
\begin{gathered}
x=r \cos (\theta) \sin (\alpha) \\
y=r \sin (\theta) \sin (\alpha) \\
z=r \cos (\alpha)
\end{gathered}
$$

where $\theta=[$ start value: step: end value $]=[0: 0.001: 2 \pi], \alpha=[$ start value: step: end value $]=$

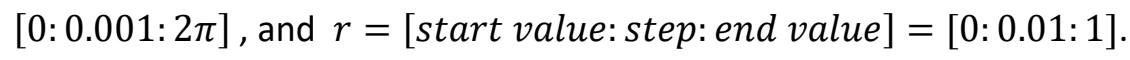

The simulation proves that $K(3)=\frac{\sqrt{2}+\sqrt{11}}{3}$.

\section{The constant $\mathrm{K}$ for dimension 4}

Before giving the approach for dimension 4, we will review the calculation for dimension 2 and 3 in a different ways.

For dimension 2, we denote by $\vec{V}_{1}=\left(\begin{array}{l}\alpha_{1} \\ \beta_{1}\end{array}\right)$ and $\vec{V}_{2}=\left(\begin{array}{l}\alpha_{2} \\ \beta_{2}\end{array}\right)$ the two particular vectors that verified

$$
\min _{\varepsilon_{1}}\left\|\vec{V}_{1}+\varepsilon_{2} \vec{V}_{2}\right\|_{\infty}=K(2) \text {. }
$$


By symmetry, we can assume that

$$
\begin{aligned}
& \left\|\vec{V}_{1}+\vec{V}_{2}\right\|_{\infty}=\alpha_{1}+\alpha_{2}=K(2) \\
& \left\|\vec{V}_{1}-\vec{V}_{2}\right\|_{\infty}=\beta_{1}-\beta_{2}=K(2)
\end{aligned}
$$

From the definition of $K(2)$, to get the maximum value of it, the coordinates of the two vectors should be non-negatives values except the coordinate $\beta_{2}$ should be a negative value.

By symmetry, we denote $\alpha_{1}=\alpha_{2}=\alpha \& \beta_{1}=-\beta_{2}=\beta$.

to find $K(2)$, it is a enough to solve the following system:

$$
\left\{\begin{array}{l}
2 \alpha=K(2) \\
2 \beta=K(2)
\end{array}\right.
$$

under the constraint $\alpha^{2}+\beta^{2} \leq 1$.

The maximum $K(2)$ can be found by considering $\alpha^{2}+\beta^{2}=1$, then the previous system is equivalent to the following equation

$$
\left(\frac{K(2)}{2}\right)^{2}+\left(\frac{K(2)}{2}\right)^{2}=1
$$

Therefore

$$
K(2) \geq \sqrt{2}
$$

For dimension 3 , we would like to find $\vec{V}_{1}=\left(\begin{array}{l}\alpha_{1} \\ \beta_{1} \\ \gamma_{1}\end{array}\right), \vec{V}_{2}=\left(\begin{array}{l}\alpha_{2} \\ \beta_{2} \\ \gamma_{2}\end{array}\right)$ and $\vec{V}_{3}=\left(\begin{array}{l}\alpha_{3} \\ \beta_{3} \\ \gamma_{3}\end{array}\right)$ that verify

$$
\min _{\varepsilon_{1}, \varepsilon_{2}}\left\|\vec{V}_{1}+\varepsilon_{2} \vec{V}_{2}+\varepsilon_{1} \vec{V}_{1}\right\|_{\infty}=K(3)
$$

We need to investigate all possible cases of the vector $\vec{\varepsilon}=\left(\begin{array}{c}\varepsilon_{1} \\ \varepsilon_{2} \\ 1\end{array}\right)$ under a matrix A, where its rows $r_{i}$ form all cases $\vec{\varepsilon}$. The matric $A_{3}$ is defined as follows:

$$
A_{3}=\left[\begin{array}{ccc}
+1 & +1 & +1 \\
+1 & -1 & +1 \\
+1 & +1 & -1 \\
+1 & -1 & -1
\end{array}\right]
$$

The four rows are not independent vectors because it is noted that $r_{4}=r_{2}+r_{3}-r_{1}$.

By symmetry, we can assume the following equations

$$
\begin{aligned}
& \left\|\vec{V}_{3}+\vec{V}_{2}+\vec{V}_{1}\right\|_{\infty}=\left|\alpha_{3}+\alpha_{2}+\alpha_{1}\right|=K(3) \\
& \left\|\vec{V}_{3}+\vec{V}_{2}-\vec{V}_{1}\right\|_{\infty}=\left|\beta_{3}+\beta_{2}-\beta_{1}\right|=K(3) \\
& \left\|\vec{V}_{3}-\vec{V}_{2}+\vec{V}_{1}\right\|_{\infty}=\left|\gamma_{3}-\gamma_{2}+\gamma_{1}\right|=K(3)
\end{aligned}
$$




$$
\left\|\vec{V}_{3}-\vec{V}_{2}-\vec{V}_{1}\right\|_{\infty}=\left|\gamma_{3}-\gamma_{2}-\gamma_{1}\right|=K(3)
$$

In order to maximize the value of (3), it is suitable to consider $\beta_{1}$ and $\gamma_{2}$ as a negative values, then the coordinate of the three vectors $\vec{V}_{i}$ will be summarized as following

$$
\vec{V}_{1}=\left(\begin{array}{c}
\alpha_{1} \\
-\beta_{1} \\
\gamma_{1}
\end{array}\right), \vec{V}_{2}=\left(\begin{array}{c}
\alpha_{2} \\
\beta_{2} \\
-\gamma_{2}
\end{array}\right) \text { and } \vec{V}_{3}=\left(\begin{array}{l}
\alpha_{3} \\
\beta_{3} \\
\gamma_{3}
\end{array}\right) \text {, }
$$

Where all parameters, $\left(\alpha_{i}, \beta_{i}, \gamma_{i}\right)$ are non-negative values.

To calculate $\mathrm{K}(3)$, it is enough to solve the below system:

$$
\left\{\begin{array}{c}
\alpha_{3}+\alpha_{2}+\alpha_{1}=K(3) \\
\beta_{3}+\beta_{2}+\beta_{1}=K(3) \\
\gamma_{3}+\gamma_{2}+\gamma_{1}=K(3) \\
-\gamma_{3}+\gamma_{2}+\gamma_{1}=K(3)
\end{array}\right.
$$

Under the constraints

$$
\begin{aligned}
& \alpha_{1}{ }^{2}+\beta_{1}{ }^{2}+\gamma_{1}{ }^{2} \leq 1 \\
& \alpha_{2}{ }^{2}+{\beta_{2}}^{2}+\gamma_{2}{ }^{2} \leq 1 \\
& \alpha_{3}{ }^{2}+\beta_{3}{ }^{2}+\gamma_{3}{ }^{2} \leq 1
\end{aligned}
$$

From the last two equations of the system we can conclude that $\gamma_{3}=0$.

By symmetry also, we can assume that

$$
\begin{aligned}
\alpha_{1}=\alpha_{2} & =\beta_{2}=\beta_{1}=\beta \\
\gamma_{2} & =\gamma_{1}=\gamma \\
\alpha_{3} & =\beta_{3}=\alpha
\end{aligned}
$$

Therefore,

$$
\left\{\begin{array}{c}
2 \beta+\alpha=K(3) \\
2 \gamma=K(3)
\end{array}\right.
$$

Under the constraints

$$
\begin{gathered}
2 \beta^{2}+\alpha^{2} \leq 1 \\
2 \alpha^{2} \leq 1
\end{gathered}
$$

In order to maximize the value of $K(3)$, the two constraints can be considered as

$$
2 \beta^{2}+\alpha^{2}=1
$$




$$
2 \alpha^{2}=1
$$

Then the system will be simplified as follows

$$
\left\{\begin{array}{c}
2 \beta=K(3)-\frac{\sqrt{2}}{2} \\
2 \gamma=K(3)
\end{array}\right.
$$

The below quadratic equation is needed to be solved to calculate the value $K(3)$,

$$
2\left(\frac{K(3)}{2}-\frac{\sqrt{2}}{4}\right)^{2}+\left(\frac{K(3)}{2}\right)^{2}=1
$$

After simplification, we got

$$
3 K(3)^{2}-2 \sqrt{2} K(3)-3=0
$$

Finally, we can conclude with simulation that

$$
K(3)=\frac{\sqrt{2}+\sqrt{11}}{3}
$$

The particular vectors that cannot canceled each other further than $K(3)$ are define as follows:

$$
\vec{V}_{1}=\left(\begin{array}{c}
\frac{K(3)}{4}-\frac{\sqrt{2}}{4} \\
\frac{-K(3)}{4}+\frac{\sqrt{2}}{4} \\
\frac{K(3)}{2}
\end{array}\right), \vec{V}_{2}=\left(\begin{array}{c}
\frac{K(3)}{4}-\frac{\sqrt{2}}{4} \\
\frac{K(3)}{4}-\frac{\sqrt{2}}{4} \\
-\frac{K(3)}{2}
\end{array}\right) \text { and } \vec{V}_{3}=\left(\begin{array}{c}
\frac{\sqrt{2}}{2} \\
\frac{\sqrt{2}}{2} \\
0
\end{array}\right) \text {. }
$$

Note that these particular three vectors are not unique that verify $\min _{\varepsilon_{i}}\left\|\sum \varepsilon_{i} \vec{V}_{i}\right\|_{\infty}=K(3)$.

Our idea is to generalize the previous approach in evaluating the constant $K$, for that let denote by $V=\left\{\vec{V}_{4}, \vec{V}_{3}, \vec{V}_{2}, \vec{V}_{1}\right\}$ as a set of particular vectors that satisfy the below equation

$$
K(4)=\min _{\varepsilon_{1}, \varepsilon_{2}, \varepsilon_{3}}\left\|\vec{V}_{4}+\sum_{i=1}^{3} \varepsilon_{i} \vec{V}_{i}\right\|_{\infty} .
$$

The matrix $A_{4}$ can be extended to fit the dimension 4, where its rows, $r_{i}$, are all possible values of $\left(1, \varepsilon_{1}, \varepsilon_{2}, \varepsilon_{3}\right)$ as follows: 


$$
A_{4}=\left[\begin{array}{llll}
+1 & +1 & +1 & +1 \\
+1 & -1 & +1 & +1 \\
+1 & +1 & -1 & +1 \\
+1 & -1 & -1 & +1 \\
+1 & +1 & +1 & -1 \\
+1 & -1 & +1 & -1 \\
+1 & +1 & -1 & -1 \\
+1 & -1 & -1 & -1
\end{array}\right]
$$

where it is noted that $r_{i+2}=r_{i+1}+r_{i}-r_{i-1}$, for $i>1$, and $\operatorname{dim}\left(\operatorname{span}\left(r_{1}, r_{2}, r_{3}\right)\right)=3$.

The idea is to well assign each row $r_{i}$ to one of fourth dimension in order to avoid zero coordinate $\ln \vec{V}_{i}$, which it is a consequence of maximizing the value of $K(4)$, i.e., the axes where L-infinity norm of $\vec{V}_{4}+$ $\sum_{i=1}^{3} \varepsilon_{i} \vec{V}_{i}$ is located will be distributed over possible combination of $\left(1, \varepsilon_{1}, \varepsilon_{2}, \varepsilon_{3}\right)$ in a way to maximize the value $K(4)$.

The below diagram, in Figure 6, identifies which coordinate will be eliminated, being zero, when we associate two rows to same axes.

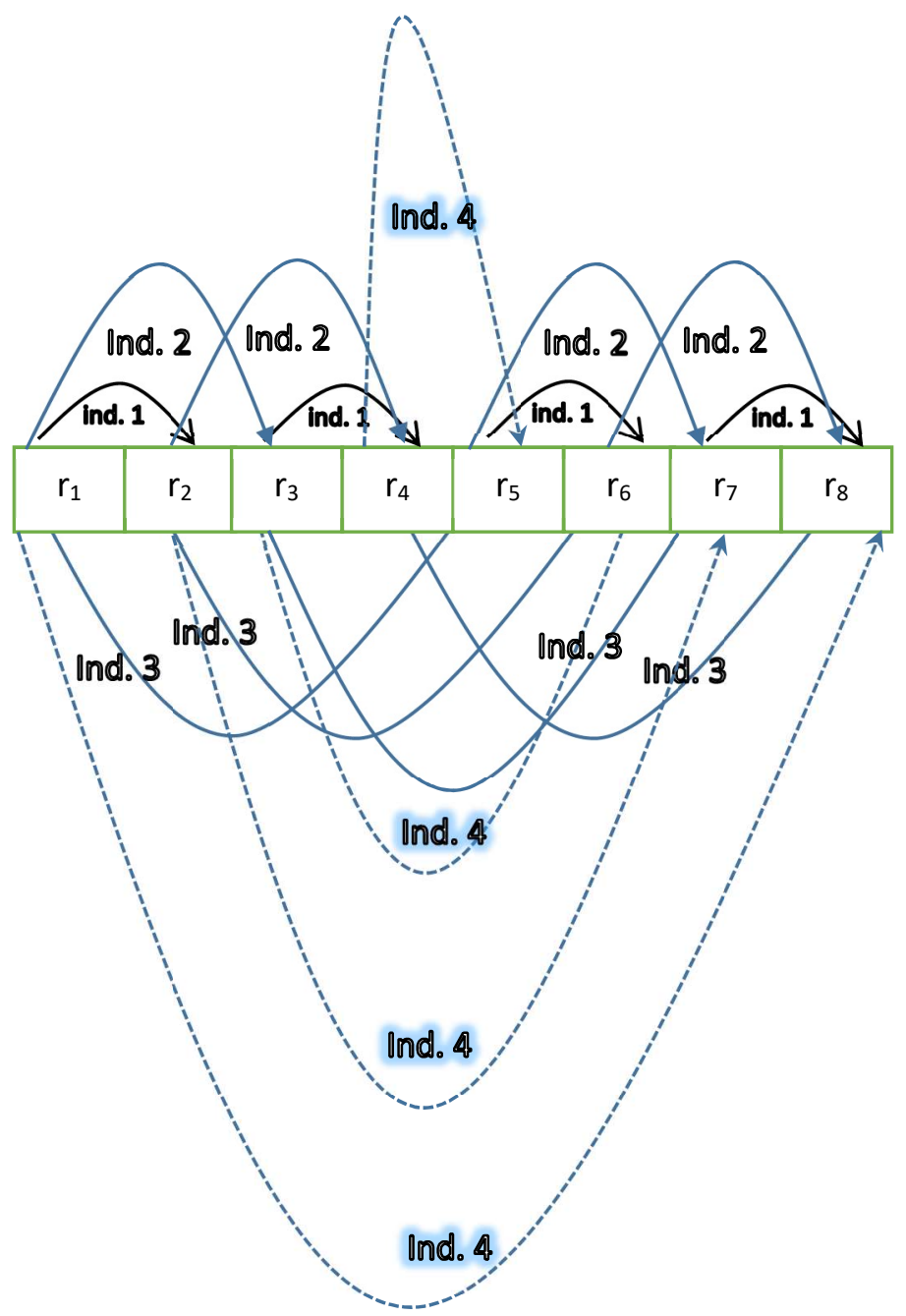

Figure 6 A link between gathering two rows as system of equation and the index variable that will be eliminated. 
From previous diagram, the rows are gathered as follows

- $\left(r_{1}, r_{2}\right):\left\|\vec{V}_{4} \pm \vec{V}_{3}+\vec{V}_{2}+\vec{V}_{1}\right\|_{\infty}=\left|V_{4}^{1} \pm V_{3}^{1}+V_{2}^{1}+V_{1}^{1}\right|$

- $\left(r_{5}, r_{7}\right):\left\|\vec{V}_{4}+\vec{V}_{3} \pm \vec{V}_{2}-\vec{V}_{1}\right\|_{\infty}=\left|V_{4}^{2}+V_{3}^{2} \pm V_{2}^{2}-V_{1}^{2}\right|$

- $\left(r_{4}, r_{8}\right):\left\|\vec{V}_{4}-\vec{V}_{3}-\vec{V}_{2} \pm \vec{V}_{1}\right\|_{\infty}=\left|V_{4}^{3}-V_{3}^{3}-V_{2}^{3} \pm V_{1}^{3}\right|$

- $\left(r_{3}, r_{6}\right):\left\| \pm \vec{V}_{4}+\vec{V}_{3}-\vec{V}_{2}+\vec{V}_{1}\right\|_{\infty}=\left| \pm V_{4}^{4}+V_{3}^{4}-V_{2}^{4}+V_{1}^{4}\right|$,

where $V_{i}^{j}$ is the j-th coordinate of vector $\vec{V}_{i}$.

From previous system of equations and to maximize the value $K(4)$ the coordinate of $\vec{V}_{i}$ will be as follows

$$
\vec{V}_{1}=\left(\begin{array}{c}
\alpha_{1} \\
-\beta_{2} \\
\gamma_{2} \\
w_{2}
\end{array}\right), \vec{V}_{2}=\left(\begin{array}{c}
\alpha_{2} \\
\beta_{2} \\
-\gamma_{2} \\
-w_{2}
\end{array}\right), \vec{V}_{3}=\left(\begin{array}{c}
\alpha_{3} \\
\beta_{3} \\
-\gamma_{3} \\
w_{4}
\end{array}\right) \text { and } \vec{V}_{4}=\left(\begin{array}{c}
\alpha_{4} \\
\beta_{4} \\
\gamma_{4} \\
w_{4}
\end{array}\right)
$$

where $\alpha_{i}, \beta_{i}, \gamma_{i}$ and $w_{i}$ are non-negative values.

The negative sign highlighted at the coordinate of $\vec{V}_{l}$ comes from rows $r_{1}, r_{5}, r_{4}$, and $r_{3}$. For instance, if we assume that $\left\|\vec{V}_{4}+\sum_{i=1}^{3} \varepsilon_{i} \vec{V}_{i}\right\|_{\infty}=\sum_{i=1}^{3}\left|\varepsilon_{i} \alpha_{i}\right|+\left|\alpha_{4}\right|$, where $r_{4}=(1,-1,-1,1)$, in order to get maximum of $K(4)$, it is preferable to consider the two coordinates $\alpha_{2}$, and $\alpha_{3}$, as negative values such that the equation $1\left(\alpha_{1}\right)-1\left(\alpha_{2}\right)-1\left(\alpha_{3}\right)+1\left(\alpha_{4}\right)=K(4)$ will be equivalent to the equation

$$
\left|\alpha_{1}\right|+\left|\alpha_{2}\right|+\left|\alpha_{3}\right|+\left|\alpha_{4}\right|=K(4) \text {. }
$$

The row distribution can formulated by the following systems of equations

$$
\begin{gathered}
\left(r_{1}, r_{2}\right):\left\{\begin{array}{l}
\alpha_{4}+\alpha_{3}+\alpha_{2}+\alpha_{1}=K \\
\alpha_{4}-\alpha_{3}+\alpha_{2}+\alpha_{1}=K
\end{array} \Rightarrow \alpha_{3}=0\right. \\
\left(r_{5}, r_{7}\right):\left\{\begin{array}{l}
\beta_{4}+\beta_{3}+\beta_{2}+\beta_{1}=K \\
\beta_{4}+\beta_{3}-\beta_{2}+\beta_{1}=K
\end{array} \Rightarrow \beta_{2}=0\right. \\
\left(r_{4}, r_{8}\right):\left\{\begin{array}{l}
\gamma_{4}+\gamma_{3}+\gamma_{2}+\gamma_{1}=K \\
\gamma_{4}+\gamma_{3}+\gamma_{2}-\gamma_{1}=K
\end{array} \Rightarrow \gamma_{1}=0\right. \\
\left(r_{3}, r_{6}\right):\left\{\begin{array}{l}
w_{4}+w_{3}+w_{2}+w_{1}=K \\
-w_{4}+w_{3}+w_{2}+w_{1}=K
\end{array} \Rightarrow w_{4}=0\right.
\end{gathered}
$$

The system can be simplified further by

$$
\left\{\begin{array}{c}
\alpha_{4}+\alpha_{2}+\alpha_{1}=K \\
\beta_{4}+\beta_{3}+\beta_{1}=K \\
\gamma_{4}+\gamma_{3}+\gamma_{2}=K \\
w_{3}+w_{2}+w_{1}=K
\end{array}\right.
$$


Under the below constraints

$$
\left\{\begin{array}{l}
\alpha_{4}^{2}+\beta_{4}^{2}+\gamma_{4}^{2}=1 \\
w_{4}^{2}+\beta_{3}^{2}+\gamma_{3}^{2}=1 \\
\alpha_{2}^{2}+w_{2}^{2}+\gamma_{2}^{2}=1 \\
\alpha_{1}^{2}+\beta_{1}^{2}+w_{1}^{2}=1
\end{array}\right.
$$

As before, the previous system of equations needs to be matched with coordinates of the four vectors in order to maximize the value of $K(4)$, then

$$
\left[\vec{V}_{1}, \vec{V}_{2}, \vec{V}_{3}, \vec{V}_{3}\right]=\left[\begin{array}{cccc}
\alpha_{1} & \alpha_{2} & 0 & \alpha_{4} \\
-\beta_{1} & 0 & \beta_{3} & \beta_{4} \\
0 & -\gamma_{2} & -\gamma_{3} & \gamma_{4} \\
w_{1} & -w_{2} & w_{3} & 0
\end{array}\right]
$$

By symmetry, we can assume that

$$
\begin{gathered}
\alpha_{4}=\alpha_{2}=\alpha_{1}=\alpha \\
\beta_{4}=\beta_{3}=\beta_{1}=\beta \\
\gamma_{4}=\gamma_{3}=\gamma_{2}=\gamma \\
w_{3}=w_{2}=w_{1}=w
\end{gathered}
$$

Therefore

$$
\left\{\begin{array}{l}
\alpha=K / 3 \\
\beta=K / 3 \\
\gamma=K / 3 \\
w=K / 3
\end{array}\right.
$$

To maximize $K$, the constraints can be assumed to be as follows:

$$
\begin{aligned}
1 & =\alpha^{2}+\beta^{2}+\gamma^{2} \\
& =\alpha^{2}+\beta^{2}+w^{2} \\
& =\alpha^{2}+w^{2}+\gamma^{2} \\
& =w^{2}+\beta^{2}+\gamma^{2}
\end{aligned}
$$

To find the value of $K(4)$, it is enough to solve the below quadrature equation

$$
\alpha^{2}+\beta^{2}+\gamma^{2}=3\left(\frac{K}{3}\right)^{2}=1
$$

It implies that

$$
K(4) \geq \sqrt{3}
$$


and the coordinates of the particular set of vectors $\vec{V}_{l}$ are summarized under the below matrix

$$
\left[\begin{array}{llll}
\overrightarrow{V_{1}} & \overrightarrow{V_{2}} & \overrightarrow{V_{3}} & \vec{V}_{4}
\end{array}\right]=\frac{1}{\sqrt{3}}\left[\begin{array}{cccc}
1 & 1 & 0 & 1 \\
-1 & 0 & 1 & 1 \\
0 & -1 & -1 & 1 \\
1 & -1 & 1 & 0
\end{array}\right] .
$$

Note: Other distribution can formulated by the following the below configuration:

The matrix can be formulated differently as follows:

$$
A_{4}=\left[\begin{array}{llll}
+1 & +1 & +1 & +1 \\
-1 & +1 & +1 & +1 \\
+1 & -1 & +1 & +1 \\
-1 & -1 & +1 & +1 \\
+1 & +1 & -1 & +1 \\
-1 & +1 & -1 & +1 \\
+1 & -1 & -1 & +1 \\
-1 & -1 & -1 & +1
\end{array}\right]
$$

From the below table, the row distribution can configurated by the following systems of equations

$$
\begin{gathered}
\left(r_{1}, r_{2}\right):\left\{\begin{array}{c}
\alpha_{1}+\alpha_{2}+\alpha_{3}+\alpha_{4}=K \\
-\alpha_{1}+\alpha_{2}+\alpha_{3}+\alpha_{4}=K
\end{array} \Rightarrow \alpha_{1}=0\right. \\
\left(r_{6}, r_{8}\right):\left\{\begin{array}{l}
\beta_{1}+\beta_{2}+\beta_{3}+\beta_{4}=K \\
\beta_{1}-\beta_{2}+\beta_{3}+\beta_{4}=K
\end{array} \Rightarrow \beta_{2}=0\right. \\
\left(r_{3}, r_{7}\right):\left\{\begin{array}{l}
\gamma_{1}+\gamma_{2}+\gamma_{3}+\gamma_{4}=K \\
\gamma_{1}+\gamma_{2}-\gamma_{3}+\gamma_{4}=K
\end{array} \Rightarrow \gamma_{3}=0\right. \\
\left(r_{4}, r_{5}\right):\left\{\begin{array}{l}
w_{1}+w_{2}+w_{3}+w_{4}=K \\
w_{1}+w_{2}+w_{3}-w_{4}=K
\end{array} \Rightarrow w_{4}=0\right.
\end{gathered}
$$

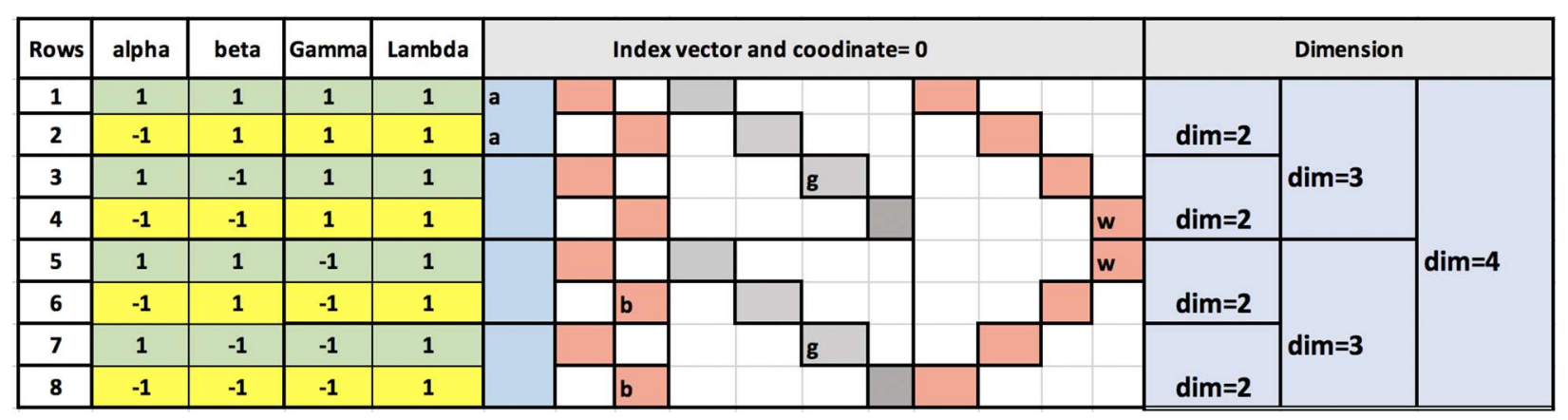

Table 1 How to gather two rows of the matrix in order to eliminate a given index coordinate.

By finishing the calculation, we find that 


$$
K(4) \geq \sqrt{3}
$$

The Cauchy-Schwarz inequality, also known as the Cauchy-Bunyakovsky-Schwarz inequality, can be used to optimized the following system:

Maximizing the variable $K$, under the fourth objective functions:

$$
\left\{\begin{array}{c}
\alpha_{4}+\alpha_{2}+\alpha_{1}=K \\
\beta_{4}+\beta_{3}+\beta_{1}=K \\
\gamma_{4}+\gamma_{3}+\gamma_{2}=K \\
w_{3}+w_{2}+w_{1}=K
\end{array}\right.
$$

Under the below constraints

$$
\left\{\begin{array}{l}
\alpha_{4}^{2}+\beta_{4}^{2}+\gamma_{4}^{2}=1 \\
w_{4}^{2}+\beta_{3}^{2}+\gamma_{3}^{2}=1 \\
\alpha_{2}^{2}+w_{2}^{2}+\gamma_{2}^{2}=1 \\
\alpha_{1}^{2}+\beta_{1}^{2}+w_{1}^{2}=1
\end{array}\right.
$$

The system can be modified by Cauchy as follows:

$$
\left\{\begin{array}{c}
K^{2}=\left(\alpha_{4}+\alpha_{2}+\alpha_{1}\right)^{2} \leq\left(\alpha_{4}^{2}+\alpha_{2}^{2}+\alpha_{1}^{2}\right)\left(1^{2}+1^{2}+1^{2}\right) \\
K^{2}=\left(\beta_{4}+\beta_{3}+\beta_{1}\right)^{2} \leq\left(\beta_{4}^{2}+\beta_{3}^{2}+\beta_{1}^{2}\right)\left(1^{2}+1^{2}+1^{2}\right) \\
K^{2}=\left(\gamma_{4}+\gamma_{3}+\gamma_{2}\right)^{2} \leq\left(\gamma_{4}^{2}+\gamma_{3}^{2}+\gamma_{2}^{2}\right)\left(1^{2}+1^{2}+1^{2}\right) \\
K^{2}=\left(w_{3}+w_{2}+w_{1}\right)^{2} \leq\left(w_{3}^{2}+w_{2}^{2}+w_{1}^{2}\right)\left(1^{2}+1^{2}+1^{2}\right)
\end{array}\right.
$$

By adding all the four equation will get

$$
4 K^{2} \leq 3\left(\alpha_{4}^{2}+\alpha_{2}^{2}+\alpha_{1}^{2}+\beta_{4}^{2}+\beta_{3}^{2}+\beta_{1}^{2}+\gamma_{4}^{2}+\gamma_{3}^{2}+\gamma_{2}^{2}+w_{3}^{2}+w_{2}^{2}+w_{1}^{2}\right),
$$

from constraints, the maximum of the value $K$ can be calculated as follows:

$$
4 K^{2}=12
$$

then the constant of the optimization is found to be as follows

$$
K=\sqrt{3},
$$

Then the komlos constant has a lower bound as follows

$$
K(4) \geq \sqrt{3} \text {. }
$$

The coordinate of the four vectors can be calculated from the equality of Cauchy-Schwarz inequality property that states that 


$$
\left\{\begin{array}{l}
\left(\alpha_{4}+\alpha_{2}+\alpha_{1}\right)^{2}=\left(\alpha_{4}^{2}+\alpha_{2}^{2}+\alpha_{1}^{2}\right)\left(1^{2}+1^{2}+1^{2}\right) \Rightarrow \frac{\alpha_{1}}{1}=\frac{\alpha_{2}}{1}=\frac{\alpha_{4}}{1} \\
\left(\beta_{4}+\beta_{3}+\beta_{1}\right)^{2}=\left(\beta_{4}^{2}+\beta_{3}^{2}+\beta_{1}^{2}\right)\left(1^{2}+1^{2}+1^{2}\right) \Rightarrow \frac{\beta_{1}}{1}=\frac{\beta_{3}}{1}=\frac{\beta_{4}}{1} \\
\left(\gamma_{4}+\gamma_{3}+\gamma_{2}\right)^{2}=\left(\gamma_{4}^{2}+\gamma_{3}^{2}+\gamma_{2}^{2}\right)\left(1^{2}+1^{2}+1^{2}\right) \Rightarrow \frac{\gamma_{2}}{1}=\frac{\gamma_{3}}{1}=\frac{\gamma_{4}}{1} \\
\left(w_{3}+w_{2}+w_{1}\right)^{2}=\left(w_{3}^{2}+w_{2}^{2}+w_{1}^{2}\right)\left(1^{2}+1^{2}+1^{2}\right) \Rightarrow \frac{w_{1}}{1}=\frac{w_{2}}{1}=\frac{w_{3}}{1}
\end{array}\right.
$$

Therefore

$$
\alpha_{i}=\beta_{i}=\gamma_{i}=w_{i}=\frac{\sqrt{3}}{3} .
$$

In the case where the dimension is under the form of $2^{m}$, for any integer $m$, the optimization is perfect but for other cases of dimension we can find upper bound of the constant $\mathrm{K}$ if Cauchy-Schwarz inequality is applied as above.

\section{The constant $\boldsymbol{K}$ for dimension $\mathbf{5}$}

By using the same idea of the previous section, dimension 4 , we denote by $\overrightarrow{V_{1}}, \ldots, \overrightarrow{V_{5}}$ as a special vector satisfying

$$
K(5)=\min _{\varepsilon_{i}}\left\|\vec{V}_{5}+\sum_{i=1}^{4} \varepsilon_{i} \vec{V}_{i}\right\|_{\infty}
$$

All the different combination of $\left(1, \varepsilon_{1}, \varepsilon_{2}, \varepsilon_{3}, \varepsilon_{4}\right)$ are summarized at the rows of the matrix $A_{5}$ defined as follows

$$
A_{5}=\left[\begin{array}{lllll}
+ & + & + & + & + \\
- & + & + & + & + \\
+ & - & + & + & + \\
- & - & + & + & + \\
+ & + & - & + & + \\
- & + & - & + & + \\
+ & - & - & + & + \\
- & - & - & + & + \\
+ & + & + & - & + \\
- & + & + & - & + \\
+ & - & + & - & + \\
- & - & + & - & + \\
+ & + & - & - & + \\
- & + & - & - & + \\
+ & - & - & - & + \\
- & - & - & - & +
\end{array}\right]
$$

where it is noted that $r_{i+2}=r_{i+1}+r_{i}-r_{i-1}$, for $i>1$ and $\operatorname{dim}\left\{r_{i}, i=1, \ldots, 16\right\}=5$. 
The target is to distribute the 16 rows among to five dimensions, named $\{\alpha, \beta, \gamma, \lambda, w\}$ in such way to minimize number of zeros in the 5 vector coordinate, $\vec{V}_{i}=\left(\begin{array}{c}\alpha_{i} \\ \beta_{i} \\ \gamma_{i} \\ \lambda_{i} \\ w_{i}\end{array}\right), i=1, \ldots, 5$.

The rows distribution is summarized as following:

- Four rows will be assigned to each axe except axe $\alpha$, where one row is a linear combination of the others $r_{i+2}=r_{i+1}+r_{i}-r_{i-1}$, it looks like each three independent rows will be assigned to one axe,

- Two rows will be assigned to axe $\alpha$

Formulating the previous distribution of the 16 rows to the below 16 equations as follows:

For $\alpha-A x e:\left\|\vec{V}_{5}+\sum_{i=1}^{4} \varepsilon_{i} \vec{V}_{i}\right\|_{\infty}=\sum_{i=1}^{4}\left|\varepsilon_{i} \alpha_{i}\right|+\left|\alpha_{5}\right|$

$$
\left\{\begin{array}{l}
r_{15}: \alpha_{5}+\alpha_{4}+\alpha_{3}+\alpha_{2}+\alpha_{1}=K(5) \\
r_{16}: \alpha_{5}+\alpha_{4}+\alpha_{3}+\alpha_{2}-\alpha_{1}=K(5)
\end{array} \Rightarrow \alpha_{1}=0\right.
$$

For $\beta$-Axe: $\left\|\overrightarrow{\boldsymbol{V}}_{\mathbf{5}}+\sum_{\boldsymbol{i}=\mathbf{1}}^{\mathbf{4}} \boldsymbol{\varepsilon}_{\boldsymbol{i}} \overrightarrow{\boldsymbol{V}}_{\boldsymbol{i}}\right\|_{\infty}=\sum_{\boldsymbol{i}=\mathbf{1}}^{\mathbf{4}}\left|\boldsymbol{\varepsilon}_{\boldsymbol{i}} \boldsymbol{\beta}_{\boldsymbol{i}}\right|+\left|\boldsymbol{\beta}_{\mathbf{5}}\right|$

$$
\left\{\begin{array}{l}
r_{1}: \beta_{5}+\beta_{4}+\beta_{3}+\beta_{2}+\beta_{1}=K(5) \\
r_{3}: \beta_{5}+\beta_{4}+\beta_{3}-\beta_{2}+\beta_{1}=K(5) \\
r_{5}: \beta_{5}+\beta_{4}-\beta_{3}+\beta_{2}+\beta_{1}=K(5) \\
r_{7}: \beta_{5}+\beta_{4}-\beta_{3}-\beta_{2}+\beta_{1}=K(5)
\end{array} \Rightarrow \beta_{2}=\beta_{3}=0\right.
$$

Note that the last equations depend on the 3 first equations.

For $\gamma$-Axe: $\left\|\vec{V}_{5}+\sum_{i=1}^{4} \varepsilon_{i} \vec{V}_{i}\right\|_{\infty}=\sum_{i=1}^{4}\left|\varepsilon_{i} \gamma_{i}\right|+\left|\gamma_{5}\right|$

$$
\left\{\begin{array}{l}
r_{2}: \gamma_{5}+\gamma_{4}+\gamma_{3}+\gamma_{2}+\gamma_{1}=K(5) \\
r_{6}: \gamma_{5}+\gamma_{4}-\gamma_{3}+\gamma_{2}+\gamma_{1}=K(5) \\
r_{10}: \gamma_{5}-\gamma_{4}+\gamma_{3}+\gamma_{2}+\gamma_{1}=K(5) \\
r_{14}: \gamma_{5}-\gamma_{4}-\gamma_{3}+\gamma_{2}+\gamma_{1}=K(5)
\end{array} \Rightarrow \gamma_{4}=\gamma_{3}=0\right.
$$

Note that the last equations depend on the 3 first equations.

For $\lambda$-Axe: $\left\|\overrightarrow{\boldsymbol{V}}_{5}+\sum_{i=1}^{4} \varepsilon_{i} \overrightarrow{\boldsymbol{V}}_{i}\right\|_{\infty}=\sum_{i=1}^{4}\left|\varepsilon_{i} \lambda_{i}\right|+\left|\lambda_{5}\right|$

$$
\left\{\begin{array}{l}
r_{4}: \lambda_{5}+\lambda_{4}+\lambda_{3}+\lambda_{2}+\lambda_{1}=K(5) \\
r_{5}: \lambda_{5}+\lambda_{4}-\lambda_{3}-\lambda_{2}-\lambda_{1}=-K(5) \\
r_{12}: \lambda_{5}-\lambda_{4}+\lambda_{3}+\lambda_{2}+\lambda_{1}=K(5) \\
r_{13}: \lambda_{5}-\lambda_{4}-\lambda_{3}-\lambda_{2}-\lambda_{1}=K(5)
\end{array} \Rightarrow \lambda_{4}=\lambda_{5}=0\right.
$$


Note that the last equations depend on the 3 first equations.

For $w$-Axe: $\left\|\vec{V}_{5}+\sum_{i=1}^{4} \varepsilon_{i} \overrightarrow{\boldsymbol{V}}_{i}\right\|_{\infty}=\sum_{i=1}^{4}\left|\varepsilon_{i} \boldsymbol{w}_{i}\right|+\left|\boldsymbol{w}_{\mathbf{5}}\right|$

$$
\left\{\begin{array}{l}
r_{6}: w_{5}+w_{4}+w_{3}+w_{2}+w_{1}=K(5) \\
r_{8}: w_{5}+w_{4}+w_{3}-w_{2}+w_{1}=-K(5) \\
r_{9}: w_{5}-w_{4}-w_{3}+w_{2}+-w_{1}=K(5) \\
r_{11}: w_{5}-w_{4}-w_{3}-w_{2}-w_{1}=K(5)
\end{array} \Rightarrow w_{5}=w_{2}=0 .\right.
$$

Note that the last equations depend on the 3 first equations.

From the previous systems of equations, we can shape our five vectors $\vec{V}_{i}$ in order to maximize $K(5)$ as follows

$$
\left[\begin{array}{lllll}
\overrightarrow{V_{1}} & \overrightarrow{V_{2}} & \overrightarrow{V_{3}} & \vec{V}_{4} & \vec{V}_{5}
\end{array}\right]=\left[\begin{array}{ccccc}
0 & -\alpha_{2} & -\alpha_{3} & -\alpha_{4} & \alpha_{5} \\
\beta_{1} & 0 & 0 & \beta_{4} & \beta_{5} \\
-\gamma_{1} & \gamma_{2} & 0 & 0 & \gamma_{5} \\
-\lambda_{1} & -\lambda_{2} & \lambda_{3} & 0 & 0 \\
-w_{1} & 0 & -w_{3} & w_{4} & 0
\end{array}\right],
$$

where $\alpha_{i}, \beta_{i}, \gamma_{i}, \lambda_{i}$, and $w_{i}$ are non-negative values.

The negative sign highlighted at the coordinate of $\vec{V}_{l}$ comes from rows $r_{15}, r_{1}, r_{2}, r_{4}$ and $r_{6}$, for example we have assume that $\left\|\vec{V}_{5}+\sum_{i=1}^{4} \varepsilon_{i} \vec{V}_{i}\right\|_{\infty}=\sum_{i=1}^{4}\left|\varepsilon_{i} \alpha_{i}\right|+\left|\alpha_{5}\right|$ where $r_{5}=(1,-1,-1,-1,1)$. Our target is to maximize the value of $K(5)$, then it is preferable to consider $\alpha_{2}, \alpha_{3}$, and $\alpha_{4}$ are negative values such that the equation $1\left(\alpha_{1}\right)-1\left(\alpha_{2}\right)-1\left(\alpha_{3}\right)-1\left(\alpha_{4}\right)+1\left(\alpha_{5}\right)=K(5)$ will be equivalent to the below equation,

$$
\left|\alpha_{1}\right|+\left|\alpha_{2}\right|+\left|\alpha_{3}\right|+\left|\alpha_{3}\right|+\left|\alpha_{5}\right|=K(5),
$$

for notation simplification notation, we write the negative parameter $\alpha_{i}$ as $-\alpha_{i}$.

To calculate the constant $K(5)$, we need to solve the below system

$$
\left\{\begin{array}{c}
\alpha_{2}+\alpha_{3}+\alpha_{4}+\alpha_{5}=K(5) \\
\beta_{1}+\beta_{4}+\beta_{5}=K(5) \\
\gamma_{1}+\gamma_{2}+\gamma_{5}=K(5) \\
\lambda_{1}+\lambda_{2}+\lambda_{3}=K(5) \\
w_{1}+w_{3}+w_{4}=K(5)
\end{array}\right.
$$

Under the constraint $\left\|\vec{V}_{i}\right\| \leq 1, i=1, \ldots, 5$.

By symmetry, we can assume the following

$$
\begin{gathered}
\alpha_{5}=\alpha_{4}=\alpha_{3}=\alpha_{2}=\alpha \\
\gamma_{5}=\gamma_{2}=\gamma \\
\lambda_{3}=\lambda_{2}=\lambda \\
w_{4}=w_{3}=w
\end{gathered}
$$




$$
\beta_{1}=\gamma_{1}=\lambda_{1}=w_{1}
$$

As $\left\|\vec{V}_{1}\right\|_{2} \leq 1$, we can put

$$
\beta_{1}=\gamma_{1}=\lambda_{1}=w_{1}=\frac{1}{2}
$$

The system will be summarized as follow:

$$
\left\{\begin{array}{c}
4 \alpha=K(5) \\
2 \beta=K(5)-\frac{1}{2} \\
2 \gamma=K(5)-\frac{1}{2} \\
2 \lambda=K(5)-\frac{1}{2} \\
2 w=K(5)-\frac{1}{2}
\end{array}\right.
$$

Under the constraints

$$
\begin{aligned}
1 & =\alpha^{2}+\beta^{2}+\gamma^{2} \\
& =\alpha^{2}+\beta^{2}+w^{2} \\
& =\alpha^{2}+\lambda^{2}+w^{2} \\
& =\alpha^{2}+\lambda^{2}+\gamma^{2}
\end{aligned}
$$

The system be equivalent to quadratic equation

$$
\left(\frac{K(5)}{4}\right)^{2}+2\left(\frac{K(5)}{2}-\frac{1}{4}\right)^{2}=1
$$

So we can conclude that the value of $K(5)$ is lower bounded by $\frac{4+\sqrt{142}}{9}$ i.e.

$$
K(5) \geq \frac{4+\sqrt{142}}{9}
$$

To see the importance of the way of distributing the rows among the axes is very important, we try to make, as an example, another configuration as follows:

$$
\begin{cases}\left(r_{7}, r_{8}, r_{9}, r_{10}\right) & \text { for } \alpha-A x e \\ \left(r_{2}, r_{3}, r_{4}\right) & \text { for } \beta-\text { Axe } \\ \left(r_{12}, r_{14}, r_{16}\right) & \text { for } \gamma-\text { Axe } \\ \left(r_{5}, r_{13}, r_{1}\right) & \text { for } \lambda-A x e \\ \left(r_{6}, r_{11}, r_{15}\right) & \text { for } w-A x e\end{cases}
$$

The five vectors coordinate will be summarized under the below matrix 


$$
\left[\begin{array}{lllll}
\overrightarrow{V_{1}}, & \overrightarrow{V_{2}}, & \overrightarrow{V_{3}}, & \vec{V}_{4}, & \vec{V}_{5}
\end{array}\right]=\left[\begin{array}{ccccc}
0 & -\alpha_{2} & -\alpha_{3} & \alpha_{4} & 0 \\
0 & 0 & \beta_{3} & \beta_{4} & \beta_{5} \\
-\gamma_{1} & 0 & 0 & -\gamma_{4} & \gamma_{5} \\
\lambda_{1} & \lambda_{2} & 0 & 0 & \lambda_{5} \\
-w_{1} & w_{2} & 0 & w_{4} & 0
\end{array}\right]
$$

The system that needs to be solved is formulated as follows:

$$
\begin{gathered}
\alpha_{2}+\alpha_{3}+\alpha_{4}=K(5) \\
\beta_{3}+\beta_{4}+\beta_{5}=K(5) \\
\gamma_{1}+\gamma_{4}+\gamma_{5}=K(5) \\
\lambda_{1}+\lambda_{2}+\lambda_{5}=K(5) \\
w_{1}+w_{2}+w_{4}=K(5)
\end{gathered}
$$

Under the constraints: $\left\|\vec{V}_{i}\right\|_{2} \leq 1$.

By using this type of distribution, the symmetry of the matrix $\left[\vec{V}_{1}, \ldots, \vec{V}_{5}\right]$ is broken, which it makes the system hard to be solve analytically and number of zero coordinate in the set of vectors $\vec{V}_{l}$ has been increased from 9 times to 10 times.

Therefore, the system that needs to be optimized is as follows:

$$
\begin{aligned}
\operatorname{Max} K(5) & =\alpha_{2}+\alpha_{3}+\alpha_{4} \\
& =\beta_{3}+\beta_{4}+\beta_{5} \\
& =\gamma_{1}+\gamma_{4}+\gamma_{5} \\
& =\lambda_{1}+\lambda_{2}+\lambda_{5} \\
& =w_{1}+w_{2}+w_{4}
\end{aligned}
$$

Under the constraints: $\left\|\vec{V}_{i}\right\|_{2} \leq 1$.

The value of $K(5)$ is very sensitive to the distribution choices, please refer the below table for different choices.

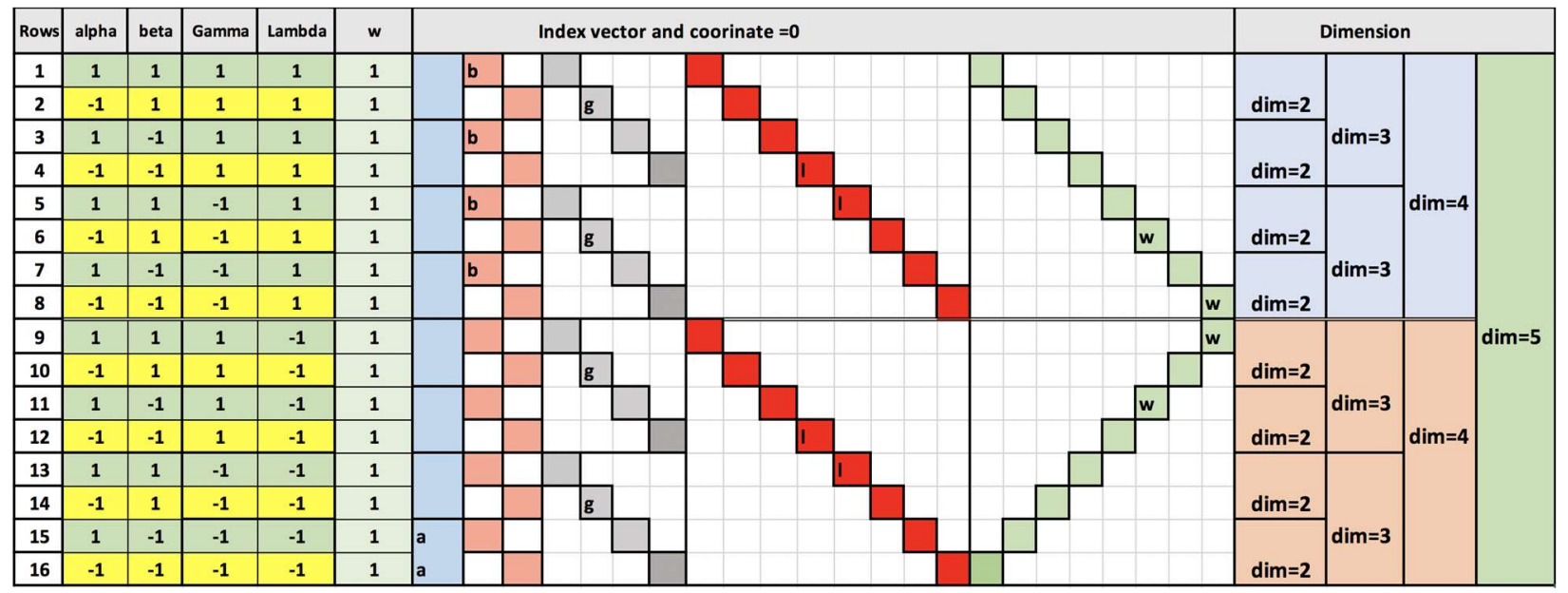

Table 2 How to gather rows of the matrix in order to eliminate a certain axes-coordinates. 


\section{Conclusion}

In dimension $n$, it is very crucial to find a best way to distribute all possible combinations of the vectors $\vec{\varepsilon}=\left(1, \varepsilon_{1}, \ldots, \varepsilon_{n-1}\right)$ among the $n$ axes. We assume that we have $\left[\frac{2^{n-1}}{n}\right\rceil$ different combinations of the vector of $\vec{\varepsilon}$ for which

$$
\left\|\vec{V}_{n}+\sum_{i=1}^{n-1} \varepsilon_{i} \vec{V}_{i}\right\|_{\infty}=\left|\sum \varepsilon_{i} x_{i}+x_{n}\right|=K(n),
$$

where $x_{i}$ is the coordinate of vector $\vec{V}_{i}$ corresponding to X-Axe.

The $\left[\frac{2^{n-1}}{n}\right\rceil$ vectors that have been assign to one axes has a dimension of order $\left\lceil\log _{2}\left(\frac{2^{n-1}}{n}\right)\right\rceil$, and as consequence, it implies that each vector $\vec{V}_{i}$ has $\left\lceil\log _{2}\left(\frac{2^{n-1}}{n}\right)\right\rceil$ null coordinate.

To evaluate the constant $K(n)$, it is enough to solve the below optimization equation

$$
\max _{x_{i}}\left(K(n)=\sum_{i=1}^{n-\left\lceil\log _{2}\left(\frac{2^{n-1}}{n}\right)\right\rceil} x_{i}\right.
$$

By imposing the symmetry conditions by choosing a good way of distribution, the non-null coordinate in each axes as constant values, i.e., $x_{i}=x$.

Let $B$ a suset $\{1, \ldots, n\}$ of cardinality around $n-\left\lceil\log _{2}\left(\frac{2^{n-1}}{n}\right)\right\rceil$ and from the condition that $\left\|\vec{V}_{n}\right\|_{2} \leq 1$, the upper bound of $x$ can be found as following

$$
\sum_{j \in B}\left(x_{i}^{j}\right)^{2}=\sum_{j \in B}(x)^{2} \leq 1 \Rightarrow x \leq \frac{\sqrt{n-\left\lceil\log _{2}\left(\frac{2^{n-1}}{n}\right)\right\rceil}}{n-\left\lceil\log _{2}\left(\frac{2^{n-1}}{n}\right)\right\rceil}
$$

The lower bound of Kmolos conjecture can be calculated as follows:

$$
\begin{aligned}
K(n) & \geq \sqrt{n-\left\lceil\log _{2}\left(\frac{2^{n-1}}{n}\right)\right\rceil} \\
& \geq \sqrt{\log (n)-1} .
\end{aligned}
$$

Under our lemma, if it exists an natural $n$ such that $n=2^{k}$, then the symmetry conditions can be used always in order to conclude that

$$
K(n)=\sqrt{n-\left\lceil\log _{2}\left(\frac{2^{n-1}}{n}\right)\right\rceil}=\sqrt{\log _{2}(n)}
$$




\section{References}

1. A. Dvoretzky, Some results on convex bodies and Banach spaces, Proceedings of the Symposium on Linear Spaces, J erusalem 1961, pp. 123-160.

2. A.Szankowski, On Dvoretzky's theorem on almost spherical sections of convex bodies, Israel J. Math. 17, (1974), 325-338.].

3. B. Chazelle. The discrepancy Method Cambridge University, Press, 1991.

4. D. Hajela, On a Conjecture of Komlos about Signed Sums of Vectors inside the Sphere . Europ. J. Combinatorics (1988) 9, 33-37.

5. J.Becka and T.Fiala. Integer-making theorem. Discrete Applied Mathematics, 3(1):1-8,1981.

6. J. Beck and V.T. Sos. Discrepancy theory. In Handbook of combinatorics (vol.2), page 1446. MIT Press, 1996

7. J. Matousek, Geometric Discrepancy. “ An Illustrated Guide, Springer Verlag 2010

8. J. Spencer. Ten lectures non the probabilistic method: second Edition. SIAM, 1994J.

9. J. Spencer, Six standard deviations suffice, Trans. Amer. Math. Soc. 289 (1985), 679-706

10. T. Figiel, Some remarks on Dvoretzky's theorem on almost spherical sections of convex bodies, Colloq. Math. 24 (1972), 241-252.

11. W. Banaszczyk . Balancing vectors and Gaussian measures of $\mathrm{n}$-dimensional convex bodies, Random structures and algorithms 12(4):351-360,1998 